\title{
Pratiques de développement territorial intégré
}

\author{
André-Anne Parent, professeure, Université de Montréal \\ René Lachapelle, stagiaire postdoctoral, Université du Québec en Outaouais \\ Denis Bourque, professeur, Université du Québec en Outaouais \\ Christian Jetté, professeur, Université de Montréal
}

\section{INTRODUCTION}

\section{Son objectif est de mieux comprendre les modes de coordination et de coopération des acteurs ainsi que les modes de fonctionnement des dispositifs de concertation à l'origine des processus de développement intégré des territoires.}

Depuis les années 1980, le développement territorial occupe une place importante en intervention collective au Québec. La création des corporations de développement communautaire, la mise en place des centres locaux de développement, l'adoption d'une stratégie de développement des communautés en santé publique, le Forum sur le développement social sont autant d'initiatives qui ont imposé la notion de territoire habité dans la réflexion et la recherche sur l'action collective. Les enjeux de développement durable ajoutent à la pertinence d'aborder le territoire habité ${ }^{1}$ en tant que lieu d'intégration des pratiques des différents acteurs qui le font exister.

\section{PROBLÉMATIQUE}

Les concertations intersectorielles seraient devenues le principal mode de résolution des problèmes complexes, soulevant cependant plusieurs questions et critiques, notamment quant à ses dérives d'hyperconcertation qui épuisent l'intersectorialité.

$\mathrm{Au}$ cours des dernières décennies, de nouveaux modèles de développement social, inspirés du concept d'État-partenaire, ont rendu possible la coconstruction de réponses aux besoins des communautés ${ }^{2,3,4}$. Durant cette période, la gestion publique a été marquée par une effervescence collaborative, à l'image de la société où priment les interactions et la connectivité générale ${ }^{5}$. Ce nouveau
La Chaire de recherche du Canada en organisation communautaire s'est intéressée à des démarches concrètes de développement territorial intégré (DTI), réalisées ou en cours au Québec. Nous cherchons à comprendre, dans le cadre du projet « Pratiques et métiers du développement territorial intégré »(CRSH 435-2013-1109), comment se concrétise la mise en œuvre de cette approche. L'équipe scientifique a débuté ses travaux en étudiant rétrospectivement les systèmes locaux d'action collective (SLA) et les modes de gouvernance à l'œuvre dans six démarches de développement prenant le territoire comme principe structurant. Cet article présente les résultats d'un stage postdoctoral effectué dans le cadre de ce projet de recherche. Son objectif est de mieux comprendre les modes de coordination et de coopération des acteurs ainsi que les modes de fonctionnement des dispositifs de concertation à l'origine des processus de développement intégré des territoires.

paradigme de développement repose principalement sur les notions de concertation/partenariat et favorise des approches de développement fondées sur des processus coopératifs. Aujourd'hui, les concertations intersectorielles seraient devenues le principal mode de résolution des problèmes complexes, soulevant cependant plusieurs questions et critiques, notamment quant à ses dérives d'hyperconcertation qui épuisent l'intersectorialité ${ }^{6}$.

À travers les mécanismes de concertation dans plusieurs domaines de l'action publique, les communautés tentent de se donner une vision cohérente du développement et des stratégies pour coordonner leurs efforts et augmenter l'efficacité des interventions ${ }^{7}$. L'approche de développement 
territorial intégré (DTI) reflète cette visée et repose sur une capacité politique à organiser des négociations entre des acteurs collectifs, à favoriser des coopérations de différentes natures susceptibles de co-construire des arrangements « viables, vivables et équitables » entre les acteurs des différents secteurs (économie, social, culture, environnement) et à produire des innovations institutionnelles pour les soutenir ${ }^{8}$.

Le principal défi du DTI concerne la mise en œuvre des réponses aux enjeux collectifs en donnant plus de pouvoir aux citoyens et aux collectivités en tant qu'acteurs de leur développement. Sur le plan du leadership, Klein identifie «trois éléments cruciaux » de l'efficacité des systèmes locaux d'action: «1) l'insertion des leaders dans de multiples réseaux de nature territoriale et sectorielle; 2) la stabilité du leadership afin de développer une connaissance des réseaux et des ressources existantes; 3) l'ouverture et la capacité de partager le savoir-faire acquis $»^{9}$. Pour Divay,

\section{MÉTHODOLOGIE}

\section{La stratégie prospective a été circonscrite à trois pratiques en émergence en recourant au partage et à l'utilisation des connaissances (PUC).}

Cette recherche qualitative est basée sur une analyse inductive de phénomènes en émergence sur le terrain. Le projet a misé dans un premier temps sur une stratégie rétrospective reposant sur une analyse de cas multiples (Yin, 1994; 1999) ${ }^{12}$ présentés dans un ensemble de monographies qui feront l'objet d'une analyse transversale ${ }^{13}$. Dans un second temps, une stratégie prospective basée sur une approche de recherche-intervention a été déployée. Dans le cadre de cette stratégie, la chercheure a veillé à enrichir la compréhension qu'ont les acteurs de leur système local d'action, et à les soutenir en partageant les connaissances acquises dans le volet rétrospectif afin d'optimiser les pratiques collaboratives ${ }^{14}$.

Réalisée dans le cadre d'un stage postdoctoral, la stratégie prospective a été circonscrite à trois pratiques en émergence en recourant au partage et à l'utilisation des connaissances (PUC) ${ }^{15,16}$. À partir de l'avancement des projets et des appren-
Belley et Prémont, la collaboration se joue sur deux plans, soit le plan de la coordination entre les acteurs et le plan de la coopération ${ }^{10}$, allant d'une entente sur l'atteinte d'objectifs communs à une harmonie et un partage complet de ressources. Cela exige toutefois un certain degré de coopération entre les acteurs qui peut prendre trois formes (Jacquier, 2008) : des coopérations horizontales afin de lutter contre la fragmentation des territoires et offrir des services qui dépassent les capacités individuelles des partenaires; une coopération verticale permettant de rompre avec une approche descendante ou hiérarchique, pour valoriser une approche ascendante voire négociée dans l'élaboration et la mise en œuvre de politiques publiques; enfin, une coopération transversale pour réduire l'approche en silo dans la mise en œuvre des politiques et la sectorisation des services et organismes qui les mettent en œuvre ${ }^{11}$. Pour Jacquier, cette dernière condition permet de passer d'une organisation de type «appareil» à une véritable organisation de type « réseau » d'acteurs.

tissages réalisés d'une rencontre à l'autre, il fut possible de rendre visible la progression de l'action collective et des apprentissages pour en dégager des connaissances praxéologiques sur le plan professionnel et organisationnel. La collecte de données fut élaborée autour de l'observation participante (Burawoy, 2000) ${ }^{17}$ lors d'événements ou de rencontres dans les milieux afin de bien comprendre leur dynamique particulière et de moments de rétroaction entre la chercheure et les participants à l'occasion de ces rencontres ou par la suite. Ces activités ont fait l'objet de notes d'observation constituant une sorte de carnet de bord de la démarche de recherche en vue du rapport final à venir.

Deux critères ont guidé le choix des pratiques à étudier. Le premier est le potentiel de pérennité qui dépend de la diversité et la représentativité des acteurs impliqués dans les pratiques en émergence. Le deuxième critère est la représentativité des milieux urbains et ruraux. Une démarche a été accompagnée dans chacun des milieux suivants: milieu rural, milieu périurbain/rural et milieu urbain. Ces trois démarches ont été accompagnées par l'équipe de recherche pendant près de deux ans, de 2013 à 2015. 
Il est important de noter que la démarche d'accompagnement a été réalisée au moment où le gouvernement du Québec opérait d'importantes transformations dans les politiques publiques. L'abolition des Conférences régionales des élus (CRÉ), la fin du Plan d'action gouvernemental pour la solidarité et l'inclusion sociale, la «nouvelle gouvernance régionale» reconnaissant aux Municipalités régionales de comté (MRC) des responsabilités de développement, notamment quant à l'existence ou non d'un Centre local de développement (CLD) sur leur territoire, la disparition de la Politique

\section{RÉSULTATS}

\subsection{Premier cas : une démarche en milieu rural}

\section{La recherche a permis de mettre au jour une stratégie plus globale de développement collectif ou le groupe de travail cherche à influencer les orientations stratégiques en développement social de la MRC.}

L'accompagnement de cette démarche a débuté suite à une présentation des chercheurs sur le sujet du développement territorial intégré. Face à une problématique d'hyperconcertation sur le territoire, un groupe de travail s'est formé, composé de deux organisateurs communautaires du CSSS, du coordonnateur de la table locale de Québec en Forme, du président du Conseil des commissaires de la Commission scolaire, du préfet suppléant de la MRC, de l'agent de développement rural du CLD et d'un représentant du Carrefour jeunesse emploi. L'objectif premier est de résoudre l'hyperconcertation sur le territoire, mais la recherche a permis de mettre au jour une stratégie plus globale de développement collectif où le groupe de travail cherche à influencer les orientations stratégiques en développement social de la MRC.

$\mathrm{Au}$ tout début, l'organisation d'un forum par la MRC préoccupe grandement le groupe de travail. La planification stratégique de la MRC requise pour le renouvèlement de la Politique nationale de la ruralité occupe un grand espace. Trois rencontres sectorielles dans la MRC, auxquelles ont participé environ 70 personnes à chaque fois, ont eu lieu, sous les thèmes de l'économie, le territoire et la société. Ces rencontres visaient à présenter un portrait de la situation et établir un diagnostic. Le groupe de nationale de la ruralité (PNR) et l'adoption du Projet de loi 10 qui a regroupé à l'échelle des régions les Centres des santé et de services sociaux (CSSS) dans les CISSS/CIUSSS, sont parmi les éléments contextuels qui eu un impact direct sur les expériences accompagnées ${ }^{18}$. Cette conjoncture impose des contraintes en même temps qu'elle offre une occasion de vérifier les critères de pérennisation que les monographies ont déjà permis d'identifier, et de les offrir aux acteurs des SLA pour l'évaluation et l'orientation de leur propre démarche.

travail cherche à identifier les éléments communs de ces rencontres pour influencer le plan d'action de la MRC. Les points de convergence identifiés sont l'identité, la communication, la concertation et la vision commune. Le forum devait ensuite permettre de dégager une vision commune du territoire. Le groupe n'a cependant pas trouvé sa place dans le processus consultatif et les membres ont été relégués à des rôles de soutien, notamment l'animation des ateliers de travail. Malgré cette marginalisation, la synthèse du forum met de l'avant certaines thématiques qui recoupent en partie les préoccupations du groupe de travail : les difficultés sur le plan des télécommunications, le besoin de mettre en place une table intersectorielle pour l'ensemble de la MRC, le désir de créer des pôles de développement et finalement, le souhait de réaliser un plan de communication pour l'ensemble de la MRC.

Au même moment, le groupe de travail effectue une analyse des tables de concertation du territoire, pour laquelle un questionnaire avait été précédemment envoyé aux responsables de ces tables. Trois constats se dégagent de l'analyse : 1) il est difficile d'arrimer les tables axées sur les clientèles et celles sur les problématiques; 2) il y a un écart entre les objectifs des tables et les besoins de la population; 3) le pouvoir d'action de certains acteurs s'est effrité depuis le retrait de l'appui du gouvernement québécois à plusieurs instances de concertation. Suite à cette analyse, un évènement est planifié pour partager les constats. L'accompagnement de notre équipe de recherche est offert pour définir les objectifs de l'évènement et les façons de les atteindre et pour faire la liaison avec des experts de 
contenu. Un suivi individuel est offert à ce sujet à l'organisateur communautaire du Centre de santé et de services sociaux (CSSS). Cela permet au groupe de travail de revoir les objectifs de mobilisation et de transfert de connaissances. On convient que le chercheur principal de l'équipe fera une présentation basée sur l'analyse des cas étudiés dans le premier volet de la recherche et abordera les rôles et objectifs de la concertation intégrée.

L'évènement a pour but de mobiliser les acteurs du milieu, les sensibiliser aux effets de la multiplication des concertations sur le territoire, susciter la réflexion sur la notion du développement territorial intégré (DTI) et présenter la concertation comme levier d'action du développement territorial. Tous les partenaires des tables de concertation sont invités; une trentaine de participants, issus majoritairement du milieu communautaire et institutionnel, ainsi que deux élus sont présents. Quatre priorités d'action se dégagent de cette journée : 1) partager l'information à tous les acteurs du milieu; 2) créer une instance avec les décideurs des institutions (CSSS, écoles, MRC, etc.); 3) travailler à partir des structures en place et mieux les utiliser; 4) mettre en place des projets porteurs pour la MRC. Ces priorités inspireront les objectifs de travail de la prochaine année, mais les participants ciblent comme première action la création d'une instance à laquelle participeront les décideurs et mandatent le groupe de travail pour la mettre en place.

Peu de temps après cet évènement, les membres du groupe de travail ont eu l'occasion de présenter ces priorités au Conseil de la MRC. Malheureusement, cela semble soulever peu d'intérêt de la part les élus qui y siègent. Suite à cette rencontre, le doute s'installe chez les membres du groupe de travail quant à la réelle prise en compte de leurs priorités par la MRC. D'autant plus qu'au cours de la même période, la planification stratégique de la MRC ainsi que le plan de développement social de la CRÉ sont publiés. Les élus ont décidé de poursuivre, malgré la fin de la CRÉ, le travail en développement social dans le cadre du plan stratégique adopté en juin 2015, mais sans lien formel avec le groupe de travail. Les membres sont surpris de constater que les orientations proposées lors du forum de novembre n'ont pas été retenues dans cette planification stratégique et se questionnent sur le rôle que pourrait y jouer le groupe de travail. À l'été suivant, l'organisateur communautaire réalise des démarches auprès de la Fondation Lucie et AndréChagnon (FLAC) et propose d'orienter la demande de façon à placer le groupe de travail comme un incontournable dans le soutien au développement des compétences, à la formation des acteurs du milieu et au réseautage, ce qui définirait un nouveau rôle pour le groupe.

\subsection{Deuxième cas : une démarche en milieu périurbain/rural}

Les premiers contacts avec ce milieu qui couvre deux MRC, ont été réalisés auprès de l'organisateur communautaire du CSSS et de l'agent de développement responsable de l'Approche territoriale intégrée (ATI) mise de l'avant par le plan d'action de la Loi visant à lutter contre la pauvreté et l'exclusion sociale (Chapitre L-7). Une équipe composée d'organisateurs communautaires du CSSS et d'agents de développement rattachés aux CLD et à la Commission scolaire a soutenu un processus de réflexion collective menant à un modèle de gouvernance partagée du développement territorial afin de limiter l'hyperconcertation, de mobiliser les organisations et de susciter la participation citoyenne. Dans le contexte des nombreux changements structurels en cours, les acteurs locaux espèrent, grâce à ce modèle, maintenir une concertation intégrée en dépit de l'abolition des CRÉ qui compromet la poursuite de la démarche d'ATI.

Cette réflexion est menée par le Conseil des partenaires qui est en fait le réseau local de services du CSSS. La première rencontre à laquelle nous assistons, est largement dominée par les discussions au sujet des changements en cours, que ce soit dans le réseau de la santé et des services sociaux (loi 10), le milieu scolaire (fusion des commissions scolaires) ou municipal (abolition des CRÉ, abolition ou transfert des CLD). L'agent de développement social de l'ATI, l'organisateur communautaire du CSSS, le directeur du CLD et un directeur adjoint du CSSS présentent le projet sous l'angle d'une gouvernance territoriale partagée et d'une harmonisation des concertations. Les acteurs présents reçoivent un peu froidement le projet. Le terme «gouvernance » passe mal et les agents de développement du territoire choisissent de parler plutôt de «capacité d'agir de toutes les organisations » et de «système de collaboration ». Le souhait de pérenniser les actions sur le territoire malgré la fin des ATI est au centre des projets. On 
craint de perdre les acquis de mobilisation des acteurs du territoire, notamment en termes de convergence des actions.

Afin de développer une approche territoriale et de réduire le nombre de concertations, les agents de développement proposent d'harmoniser les trois tables qui s'occupent de la jeunesse. Le travail d'harmonisation du secteur jeunesse est soutenu par un conseiller et s'inspire de l'expérience d'autres démarches similaires dont COSMOSS dans le BasSaint-Laurent ${ }^{19}$.

Tout au cours de ces démarches, les agents de développement se questionnent sur leur rôle et sa reconnaissance par les autres acteurs du milieu. $\mathrm{La}$ direction du CLD désire harmoniser le leadership territorial en matière de développement économique et social tandis que d'autres partenaires, notamment le CSSS et la commission scolaire, collaborent de façon variable aux travaux d'harmonisation. À certains moments, les institutions se montrent ouvertes et désireuses de partager un certain leadership, tandis qu'à d'autres, elles se recentrent sur leur mission sectorielle et leur fonctionnement. La situation est difficile pour les agents de développement alors que des difficultés financières pointent à l'horizon avec la disparition de certains programmes de financement des organismes qui les emploient. Il est difficile dans ce contexte d'élaborer de nouveaux projets et la fin de multiples programmes suscite un sentiment d'urgence.

\section{La direction du CLD désire harmoniser le leadership territorial en matière de développement économique et social tandis que d'autres partenaires, notamment le \\ CSSS et la commission scolaire, collaborent de façon variable aux travaux d'harmonisation.}

En mai 2015, les acteurs de l'ATI préparent une demande de financement, mais il n'y a pas, à ce moment, unanimité des acteurs sur l'orientation et la forme que pourrait prendre le travail en commun. D'une part, les directeurs généraux des institutions (CIUSSS, commission scolaire, etc.) cherchent à se rencontrer entre eux. D'autre part, les élus ont l'impression de se faire imposer une structure de gouvernance partagée. S'ils croient au travail des ATI dans la lutte à la pauvreté, ils ont de la difficulté à comprendre le rôle qu'ils y jouent, et ils éprouvent des réticences face au modèle de Conseil des partenaires imposé par le CIUSSS.

Une seconde rencontre du Forum des partenaires en juin 2015 entraîne un changement de cap. La direction générale du CSSS qui occupe un nouveau poste dans la structure du CIUSSS tout en demeurant répondant pour le territoire, présente les nouvelles orientations de l'établissement. Le document qui a pour thème le «leadership partagé » est marqué par les structures et intérêts du CIUSSS. La notion de responsabilité populationnelle est placée en priorité et la concertation est définie selon les programmes clientèles de l'établissement. Trois structures sont proposées: une assemblée des partenaires une fois par an, un comité stratégique et les tables de concertation rattachées aux programmes clientèles. À prime abord, les organismes communautaires ne sont représentés ni au comité stratégique ni aux tables de concertation, sauf s'ils ont une délégation aux tables rattachées au CIUSSS. L'incompréhension est palpable lors de la présentation.

Les agents de développement de l'ATI profitent de l'occasion pour présenter le «projet pérennité » élaboré au cours des dernières semaines, porté par les MRC et appuyé par l'ensemble des partenaires de l'ATI. L'objectif est de «Conserver les acquis structurants de l'ATI et poursuivre les chantiers en cours, dans une perspective de développement durable (ou pérenne) ancrée dans les territoires ». On propose de poursuivre le travail sur les enjeux priorisés à l'automne 2014: 1) harmonisation des leaderships, des initiatives de développement et des concertations; 2) accès facilité à une nourriture de qualité en quantité suffisante, à des logements sains et abordables, aux transports, à des emplois qui permettent de sortir de la pauvreté, et aux loisirs; 3) un plus grand soutien à la persévérance scolaire.

Le «projet pérennité » est porté par les MRC de Charlevoix et de Charlevoix-Est qui ont intégré le personnel de leur CLD dont les agents de développement social de l'ATI. Une demande de financement a été déposée et acceptée par les Alliances pour la solidarité, un mécanisme régional issu du Plan d'action gouvernemental pour la 
solidarité et l'inclusion sociale. Les MRC du territoire se sont engagées pour la suite à assumer ensemble $25 \%$ des coûts sur trois ans et ont adressé une proposition de collaboration à la FLAC $(50 \%)$, à Centraide (12,5\%) et au CIUSSS (12,5\%).

Selon l'agent de développement social intégré (DSI), l'Assemblée ATI est désormais déterminante en développement territorial alors que le Conseil des partenaires s'oriente vers des enjeux sanitaires. Le contexte d'austérité et la loi 10 ont créé plus de rigidité au CIUSSS qui lie le développement des communautés à une approche par programmes. Le mandat de lutte à la pauvreté et de DSI du territoire permet d'aborder l'ensemble des enjeux et facilite le travail avec les MRC. Il y a donc désormais une distinction claire entre l'assemblée ATI et le Forum des partenaires du CIUSSS. Le développement social devient une responsabilité des MRC qui souhaitent par ailleurs continuer à travailler avec le CIUSSS. À partir de ce moment, les agents de DSI ont le sentiment d'une plus grande liberté d'action dans leur travail avec les élus et avec l'ensemble des partenaires. Le leadership du milieu passe du réseau de la santé et des services sociaux aux MRC.

\subsection{Une démarche en milieu urbain}

L'accompagnement de la démarche a débuté par des contacts informels avec le coordonnateur de la Table de quartier et l'organisateur communautaire du CSSS. Une participation à un forum ouvert a permis de comprendre que la Table a entrepris un processus de redéfinition, ce qui constitue un contexte favorable à un accompagnement. En fait, la Table se définit comme un lieu de réflexion, d'échange et d'action pour les groupes communautaires et les citoyens qui partagent le désir d'améliorer la qualité de vie dans le quartier. Par diverses initiatives et participations, elle cherche à favoriser la mixité sociale, la participation citoyenne, le sentiment d'appartenance et l'appropriation des espaces publics du quartier par les citoyens. Trois axes de travail sont privilégiés : 1) favoriser la mixité sociale dans le quartier; 2) développer et animer des espaces publics et citoyens; 3) favoriser la concertation et la collaboration d'acteurs variés du milieu. Au moment de la rencontre, la Table envisageait d'adopter une orientation de type « développement des communautés », qui reste encore à définir.
La Table de quartier est fort intéressante pour ses capacités d'animation citoyenne et de participation sociale avec les divers acteurs du milieu, quoiqu'il n'y ait pas de représentation intersectorielle à ce stade. La Table accorde la priorité aux citoyens et aux organismes communautaires dans ses processus décisionnels élargis (assemblées générales, etc.), y invitant toutefois des représentants du milieu institutionnel à participer aux réflexions avec les autres partenaires locaux. Elle maintient de nombreux liens avec ses partenaires institutionnels et travaille étroitement avec eux autour de projets concrets (fêtes, consultations, etc.). L'équipe de travail de la Table considère que l'organisation d'un forum permettrait de consolider les orientations du groupe par rapport au développement des communautés. En janvier 2015, une rencontre du comité organisateur du forum revoit l'orientation du projet de développement des communautés et choisit plutôt de travailler à susciter une plus grande participation des citoyens dans les activités du quartier plutôt que d'organiser un forum. L'accompagnement change alors d'orientation et mise sur la participation aux lieux décisionnels, l'observation et le transfert de connaissances lorsque cela s'avère pertinent.

Le contexte d'austérité qui a mené à des coupures majeures en santé publique, a des impacts sur l'organisme, car la source de financement la plus importante provient de la Direction régionale de santé publique.

Les finances sont un sujet de préoccupation récurrent et une source d'anxiété pour les employés de la Table. Le contexte d'austérité qui a mené à des coupures majeures en santé publique, a des impacts sur l'organisme, car la source de financement la plus importante provient de la Direction régionale de santé publique. À partir du moment où les coupures en santé publique sont annoncées, un climat d'insécurité règne dans l'organisme, d'autant plus que peu d'information circule sur la façon dont ces coupures seront mises en œuvre. Quelques mois plus tard, on apprend qu'il y aura une coupe de quelques milliers de dollars et aucune garantie de financement pour l'année suivante. 
Malgré tout, les activités auprès des citoyens se poursuivent et des moments d'échange ont lieu, notamment pour entendre le point de vue des citoyens du quartier sur la collaboration de l'organisme à la révision des modes d'intervention policière. Une tournée des organismes et lieux de rassemblement du milieu a lieu pour inciter les citoyens à participer aux consultations de la ville dans le cadre du plan particulier d'urbanisme dont les formulaires sont disponibles seulement en ligne. Un employé les remplit à la main avec les personnes rencontrées, dont plusieurs vivent diverses difficultés, notamment des problèmes de santé mentale.

$\mathrm{Au}$ printemps, un comité est mis en place, composé d'employés de l'organisme, de citoyens, de représentants d'organismes communautaires et de commerçants du quartier, pour organiser une grande fête sur le parvis de l'église. L'organisation de cette fête est réalisée sous un mode de fonctionnement hautement participatif qui caractérise la Table. Un employé anime la fête avec, entre autres, des photos et des cartes dessinées pour visualiser le secteur. Les participants échangent ouvertement des idées sur l'animation et les spectacles. Très convivial, l'évènement permet d'exprimer ses idées. L'inclusion est de mise. On discute principalement des aspects organisationnels dont la nourriture qui est commanditée par un commerce du quartier. Il règne une dynamique conviviale entre les personnes présentes, que ce soit citoyens, commerçants ou organismes communautaires, où chacun a de l'espace pour s'exprimer. On sent un grand respect pour les citoyens de la part des commerçants présents et des organismes communautaires. Cette ambiance est caractéristique des actions de la table qui met de l'avant la participation des citoyens, plaçant les personnes exclues des sphères de décision au cœur de sa prise de décision.

\subsection{Analyse transversale des trois cas accompagnés}

Selon notre analyse, les processus coopératifs principalement en jeu dans les démarches accompagnées sont les suivants :

1) Dans le milieu périurbain/rural, le processus est à la fois horizontal (impliquant deux MRC) et vertical, car la problématique semble être l'éloignement d'une perspective de DTI, avec l'abandon de la gouvernance partagée et le retour en force des dossiers thématiques, ainsi que l'atomisation du développement (et des agents de développement) autour de deux pôles, soit celui de la santé et des services sociaux (conseil des partenaires et autres tables, notamment la jeunesse) et celui du développement social autour des MRC et des assemblées ATI. D'un côté, on s'assure d'une organisation de services principalement associée à la mobilisation des tables de concertation, et de l'autre, on tente de mettre en place une approche « négogène ${ }^{20}$ » dans la mise en œuvre des programmes publics, particulièrement en ce qui a trait au développement social. Le leadership régional semble donc se partager entre le développement territorial mené par les MRC et la concertation sociosanitaire structurée autour du CIUSSS dont le territoire couvre toute la région. Cette situation tend ainsi à fragiliser l'objectif de perspective globale du DTI et fait croître les possibilités d'actions parallèles des deux instances.

\section{Le leadership régional semble donc se partager entre le développement territorial mené par les MRC et la concertation sociosanitaire structurée autour du CIUSSS dont le territoire couvre toute la région.}

2) Dans le milieu rural, le processus est de nature transversale malgré la faible représentation des divers secteurs d'activités. L'intervention porte sur le territoire de la MRC, quoique les transformations dans le réseau de la santé et des services sociaux aient reconfiguré le territoire du CISSS à l'échelle de la région. La démarche ne regroupe pas d'organisme à vocation économique ni environnementale. Pendant la période d'observation, les objectifs et les moyens à mettre en place restaient à définir, l'intégration du développement territorial étant un but à plus long terme. La démarche ne semble pas avoir d'influence sur la MRC ou les autres institutions publiques pour revoir la mission ou la contribution des ces organisations au développement territorial. Elle ne bénéficie pas à proprement parler de soutien financier ni d'un mandat 
reconnu qui lui donnerait légitimité et crédibilité. Les intervenants qui y participent sont employés par des organismes financés en fonction de leur mission. La problématique centrale de cette démarche demeure la reconnaissance et la légitimité de l'acteur collectif, d'une part dans le système local d'action au niveau du développement social, et d'autre part, dans l'ensemble du développement territorial. À l'instar $\mathrm{du}$ cas précédent (milieu périurbain/rural), le leadership concernant le développement territorial reste divisé et ne permet pas une mise en commun des préoccupations de l'ensemble des acteurs représentés par les diverses instances.

3) Dans le milieu urbain, le processus est de nature transversale, fruit de coopérations entre la Table de quartier et les autorités locales. Le principal objectif est de faire entendre la voix de personnes exclues dans un processus de

\section{CONCLUSION}

\section{Tout au cours de la période, la question du leadership est ressortie comme un élément majeur qui influence les autres facettes du développement.}

Il est possible d'identifier un certain nombre de déterminants communs aux démarches étudiées : le besoin de clarifier et d'affirmer le leadership pour mener à bien des actions; la nécessité d'injecter des ressources, en particulier financières; la diversification des secteurs représentés par les partenaires; l'établissement de liens de confiance entre les partenaires; le besoin de clarifier les rôles des partenaires, particulièrement les élus et les gestionnaires des institutions; et enfin, la reconnaissance même des démarches par les décideurs locaux. Au cours de l'accompagnement qui se poursuit jusqu'à la fin de 2016 par un autre stagiaire postdoctoral, ces éléments pourraient être validés et bonifiés par les acteurs locaux impliqués.

Tout au cours de la période, la question du leadership est ressorti comme un élément majeur qui influence les autres facettes du développement. S'il est important de distinguer les rôles de chacun, il semble primordial de questionner la composante institutionnelle des démarches observées. Dans revitalisation générateur d'exclusion sociale par le passé. N'intégrant pas d'institutions publiques dans ses processus décisionnels, il y a peu de tension au sein de l'organisme, les valeurs s'alignant aisément et les capacités de représentation et de participation citoyenne dans les lieux décisionnels de la Table sont importantes. Cette dernière adopte une attitude coopérative qui vise à influencer les choix, particulièrement ceux de la municipalité au regard de l'aménagement urbain. Néanmoins, la Table ne bénéficie d'aucun financement récurrent, ce qui la fragilise et la place à risque de redéfinir sa mission en fonction des orientations des bailleurs de fonds, ce qui mettrait en relief des enjeux de coopération verticale. De plus, l'absence de partenaires institutionnels affaiblit sa capacité de mobilisation au-delà des partenaires déjà cooptés et pourrait témoigner de certaines craintes quant à la cohésion d'une plus vaste coalition.

deux des trois territoires, le leadership institutionnel n'a pas favorisé le système local d'action concertée. Les transformations déjà évoquées des politiques publiques ont été des éléments contextuels qui ont modifié le rôle joué par les institutions. Ces éléments ont amené les partenaires institutionnels à se centrer sur leur mission et à se désinvestir d'une approche concertée. Il est également possible que la diminution de la place accordée au développement des communautés en santé publique ait eu un effet.

Les facteurs contextuels associés au contexte d'austérité au cours de la dernière année ont largement impacté la synergie partenariale de deux des trois démarches étudiées. Il sera intéressant dans la suite de l'accompagnement de ces démarches pendant au moins une autre année d'observer les capacités de résilience des milieux et les éléments qui favoriseront ou non le développement territorial intégré. La reconfiguration des systèmes locaux d'action sera éclairante pour l'objet de la recherche, à savoir les pratiques et métiers du développement territorial intégré. Celles et ceux qui pratiquent ces métiers sont en effet confrontés au défi d'un recadrage de leurs pratiques aussi bien dans le réseau de la santé et des services sociaux que dans le milieu municipal. 


\section{BIBLIOGRAPHIE ET NOTES}

${ }^{1}$ Caillouette, J., Dallaire, N., Boyer, G., Gagnon, S. (2007). «Territorialité, action publique et développement des communautés ", Économie et Solidarités, vol. 38(1), 8-23.

${ }^{2}$ Vaillancourt, Y. (2014). Note de recherche sur l'apport de l'économie sociale et solidaire dans la coconstruction démocratique des politiques publiques : réflexions ancrées dans les expériences canadiennes, québécoises et latino-américaines, CRISES et LAREPPS, UQÀM - Les Cahiers du CRISES, ET1406, $69 \mathrm{p}$.

${ }^{3}$ Savard, S., Bourque, D., Lachapelle, R. (2015). "Third Sector Organizations in Québec and the New Public Action in Community Development", Revue canadienne de recherche sur les OSBL et l'économie sociale, vol. 6(2), Automne, 28-41.

${ }^{4}$ Jetté, C. (2008). Les organismes communautaires et la transformation de l'État-providence. Trois décennies de coconstruction des politiques publiques dans le domaine de la santé et des services sociaux, Québec, PUQ, 422 p. ${ }^{5}$ Divay, G., Belley, S., Prémont, M.-C. (2013). « La collaboration intersectorielle: spécificités, questionnements et perspectives. Introduction : présentation du thème et des contributions à ce numéro spécial », $L a$ revue de l'innovation : la revue de l'innovation dans le secteur public (En ligne), vol. 18(2), article 1.

${ }^{6}$ Bourque (2008) définit l'hyperconcertation par «l'absence d'une régulation d'ensemble des processus de concertation intersectorielle [qui] fait en sorte qu'ils se développent souvent par sédimentation et superposition des processus et instances mobilisant les mêmes acteurs qui travaillent souvent chaque dossier de manière cloisonnée $[\ldots]$ cela crée sur le terrain une dynamique d'épuisement de l'intersectorialité ». Bourque, D., Concertation et partenariat : entre levier et piège du développement des communautés, Québec, Presses de l'Université du Québec. p. 68.

${ }^{7}$ Aubin, J.-F. (2007). « Les démarches de revitalisation intégrée, un nouveau défi pour le travail social », Revue Intervention, no 126, 127-135.

${ }^{8}$ Jacquier, C. (2008). Crises, développement soutenable et modes de régulation. Quelques enseignements européens. Conférence internationale Initiatives des communautés, politiques publiques et État social au Sud et au Nord : Les défis de la prochaine décennie Université du Québec en Outaouais 24 et 25 septembre 2008.

${ }^{9}$ Klein, J.L. (2011). « Économie sociale et territoire en contexte de mondialisation. Le développement par l'initiative locale ", dans Bellemare, G. et Klein, J.L., 2011, (dir.). Innovation sociale et territoire. Convergences théoriques et pratiques, Presses de l'Université du Québec, p.183.

${ }^{10}$ Divay, G., Belley, S., Prémont, M.-C. (2013). Op. cité.
${ }^{11}$ Jacquier, C. (2008). Op.cité.

${ }^{12}$ Yin Robert K. (1994). Case Study Research: Design and Methods, 2d ed., Thousand Oaks, CA: Sage Publications, 240 p.; Yin Robert K. (1999). "Enhancing the Quality of Case Studies in Health Services Research", Health Services Research, 34:5 Part II, pp.1209-1224.

${ }^{13}$ Les monographies sont disponibles sur le site web de la CRCOC-UQO : http://w4.uqo.ca/crcoc/.

${ }^{14}$ Bilodeau, A., Beauchemin, J., Bourque, D., Galarneau, M. (2011). L'intervention en prévention des ITSS à Montréal : potentialités, limites et défis de la collaboration dans le contexte de la création des CSSS, Chaire de recherche du Canada en organisation communautaire, Gatineau, Université du Québec en Outaouais, Cahier no 1102, $117 \mathrm{p}$.

${ }^{15}$ Denis, J.-L., Lehoux, P., Champagne, F. (2004). "A knowledge utilization perspective on fine-tuning dissemination and contextualizing knowledge", dans L. Lemieux-Charles et F. Champagne (dir.), Using knowledge and evidence in health care: Multidisciplinary perspectives (p.18-40). Toronto: University of Toronto Press.

${ }^{16}$ BOGGS J. P. (1992). "Implicit Models of Social Knowledge Use. Knowledge: Creation, Diffusion, Utilization", 14 (1), 29-62.

${ }^{17}$ Burawoy, Michael (2000), « Reaching for the global » in Burawoy, Michael, Joseph A. Blum, Sheba George, Zsuzsa Gille, Teresa Gowan, Lynne Haney, Maren Klawiter, Steven H. Lopez, Sean O Riain, Millie Thayer (2000), Global Ethnography. Forces, Connections, and Imaginations in a Postmodern World, Berkeley, Los Angeles, London: University of California Press, p.1-40. ${ }^{18}$ Le projet de loi 10 , entré en vigueur le $1^{\mathrm{er}}$ avril 2015 , a transformé le réseau public de santé et de services sociaux en faisant passer de 182 à 34 le nombre d'établissements dont 12 non fusionnés soit les centres hospitaliers universitaires (à l'exception du CHU de Sherbrooke) ainsi que les établissements du Nord.

${ }^{19} \mathrm{COSMOSS}$ est une instance régionale de concertation pour améliorer la santé et le bien-être des jeunes, de leur conception jusqu'à 30 ans. Voir : Lachapelle, R., Bourque, D. (2011). COSMOSS - Communauté ouverte et solidaire pour un monde outillé, scolarisé et en santé, Une démarche de concertation régionale en développement social, Cahier de la CRCOC no 1101, 55 p.

${ }^{20}$ L'expression est de Claudine Papin de la Coopérative Tandem et signifie que l'approche est en partie exogène parce qu'influencée par les programmes et le financement en provenance des bailleurs de fonds publics ou privés, et en partie endogène parce que reposant sur la participation et l'appropriation des acteurs locaux. 


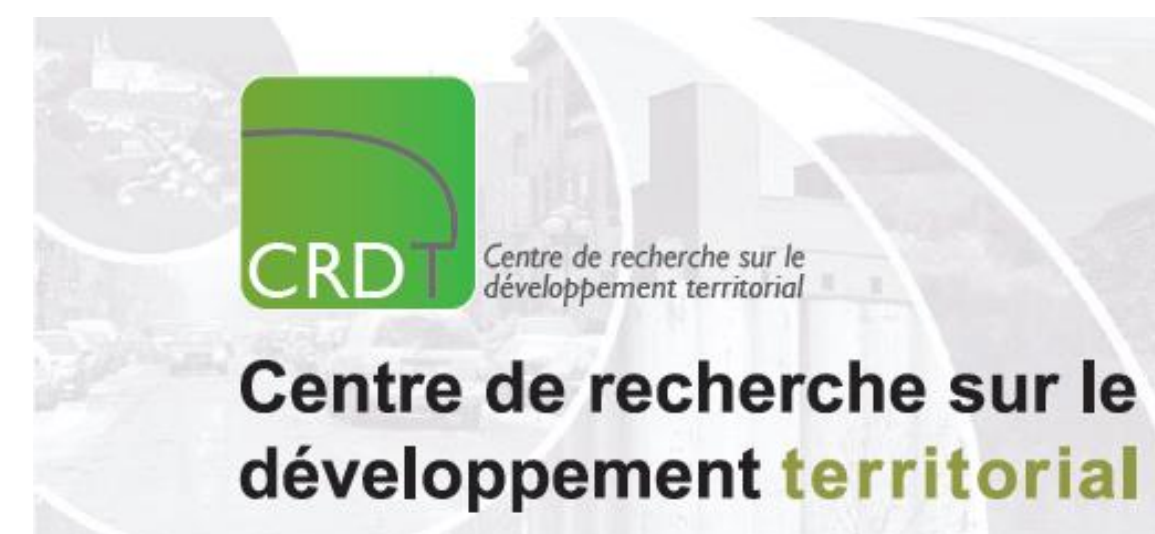

UQAC - UQAR - UQAT - UQO - INRS-UCS - ÉNAP

Le Centre de recherche sur le développement territorial (CRDT) est un regroupement stratégique de chercheurs reconnu par le Fonds québécois de recherche sur la société et la culture.

Rassemblant près d'une cinquantaine de membres, dont une dizaine de l'extérieur du Canada, le CRDT est principalement rattaché à des institutions universitaires implantées au coeur même des régions du Québec.

Le CRDT met en oeuvre une programmation intégrée de recherche comparative sur le développement territorial :

\section{AXE 1}

Aménagement et gestion durables

(1) du territoire et des ressources

\section{AXE 2}

Dynamiques économiques, production et proximité

\section{AXE 3}

Politiques publiques et gouvernance territoriale
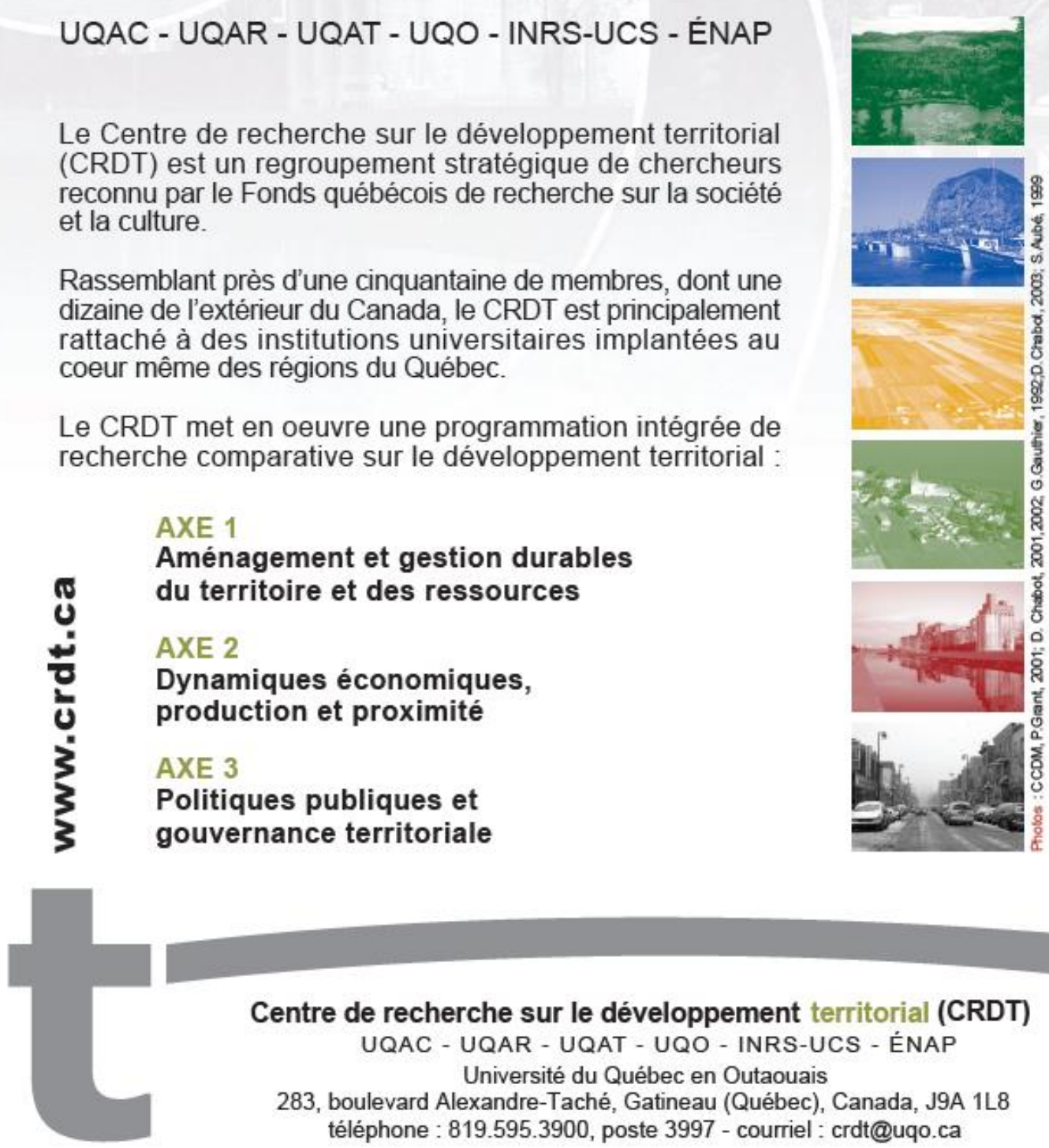

Centre de recherche sur le développement territorial (CRDT)

UQAC - UQAR - UQAT - UQO - INRS-UCS - ÉNAP

Université du Québec en Outaouais

283, boulevard Alexandre-Taché, Gatineau (Québec), Canada, J9A 1L8

téléphone : 819.595.3900, poste 3997 - courriel : crdt@uqo.ca 